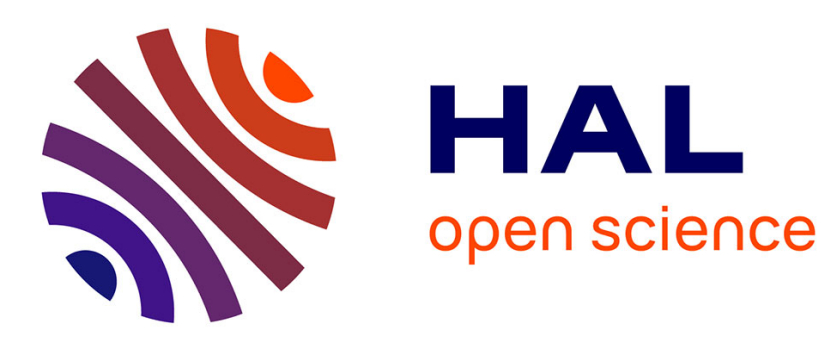

\title{
Ultrabright Silica-Coated Organic Nanocrystals for Two-Photon In Vivo Imaging
}

Shridevi Shenoi Perdoor, Fabien Dubois, Aude Barbara, Christophe Nguyen, Lamiaa M A Ali, Magali Gary-Bobo, Ines Hristovska, Kassandre Combet, Olivier Pascual, Guillaume Micouin, et al.

\section{To cite this version:}

Shridevi Shenoi Perdoor, Fabien Dubois, Aude Barbara, Christophe Nguyen, Lamiaa M A Ali, et al.. Ultrabright Silica-Coated Organic Nanocrystals for Two-Photon In Vivo Imaging. ACS Applied Nano Materials, 2020, 3 (12), pp.11933-11944. 10.1021/acsanm.0c02499 . hal-03046950

\section{HAL Id: hal-03046950 https://hal.science/hal-03046950}

Submitted on 8 Dec 2020

HAL is a multi-disciplinary open access archive for the deposit and dissemination of scientific research documents, whether they are published or not. The documents may come from teaching and research institutions in France or abroad, or from public or private research centers.
L'archive ouverte pluridisciplinaire HAL, est destinée au dépôt et à la diffusion de documents scientifiques de niveau recherche, publiés ou non, émanant des établissements d'enseignement et de recherche français ou étrangers, des laboratoires publics ou privés. 


\title{
Ultrabright Silica-Coated Organic Nanocrystals
}

\section{for Two-Photon In Vivo Imaging}

Shridevi Shenoi Perdoor, ${ }^{a}$ Fabien Dubois, ${ }^{a *}$ Aude Barbara, ${ }^{a}$ Christophe Nguyen, ${ }^{b}$ Lamiaa M. A. Ali, ${ }^{b, c}$ Magali Gary-Bobo, ${ }^{b}$ Ines Hristovska, ${ }^{d}$ Kassandre Combet, ${ }^{d}$ Olivier Pascual, ${ }^{d}$ Guillaume Micouin,,${ }^{e}$ Akos Banyasz, ${ }^{e}$ Yann Bretonnière, ${ }^{e}$ Chantal Andraud ${ }^{e}$ Alain Ibanez $^{a}$ and Xavier Cattoën, ${ }^{a^{*}}$

${ }^{a}$ Univ. Grenoble Alpes, CNRS, Grenoble INP, Institut Néel, 38000 Grenoble, France.

${ }^{b}$ IBMM, Univ Montpellier, CNRS, ENSCM, Montpellier, France.

c Department of Biochemistry, Medical Research Institute, University of Alexandria, 21561 Alexandria, Egypt

${ }^{d}$ Institut NeuroMyoGène, Equipe Synaptopathies et Autoanticorps (SynatAc), INSERM U1217/UMR CNRS 5310/University of Lyon, Université Claude Bernard Lyon 1, Lyon, France. ${ }^{e}$ Univ Lyon, ENS de Lyon, CNRS UMR 5182, Université Lyon I, Laboratoire de Chimie, F-69342 Lyon, France

\section{fabien.dubois@neel.cnrs.fr}

\section{xavier.cattoen@neel.cnrs.fr}

\begin{abstract}
Herein, we demonstrate the potential of ultrabright fluorescent silica-coated organic nanocrystals for two-photon in vivo imaging. These unique nanoparticles (NPs) containing a crystalline core of small push-pull dipolar dye are specifically designed to exhibit two-photon absorption for
\end{abstract}


fluorescence imaging. The NPs can be easily functionalized using click chemistry in pure water, with preservation of the organic core. A novel small-volume DLS technique was used to evaluate the effect of PEGylation on the colloidal stability of the NPs in complex media containing salts and proteins, mimicking the composition of blood serum. The potential of these bright red-emitting NPs for two-photon fluorescence imaging is demonstrated both in vitro and in vivo.

\section{Keywords}

Nanoparticles; bio-imaging; two-photon absorption; dynamic light scattering; colloidal stabilization

\section{Introduction}

Optical imaging techniques offer several advantages for early diagnosis of tumors and imagingguided therapy compared to alternative approaches, such as high sensitivity, high spatial resolution, high signal to noise ratio in the near infrared spectral region and the use of low concentrations of contrast agent, ${ }^{1-5}$. However, optical imaging has been primarily limited to dermal and ocular applications, due to autofluorescence, light scattering and attenuation by tissues and the absorption of light by hemoglobin, lipids and water, leading to low penetration depths. ${ }^{6,7}$ Optical imaging in the near infrared (NIR) region (650 nm - $1700 \mathrm{~nm}$ ), where light scattering and photon absorption are minimal, enables deeper tissue penetration and improved signal to noise ratios. ${ }^{6,7}$

Nonlinear optics and the development of multiphoton microscopy have further helped to achieve deeper tissue penetration, thereby expanding the scope of in vivo optical approaches for deep tissue imaging. Two-photon microscopy, which exploits the simultaneous absorption of two photons, each with half the energy (and twice the wavelength) of the corresponding single photon transition, to excite the fluorophore, enables deeper tissue penetration as it typically shifts the excitation wavelength from the visible to the biological transparency window in the NIR range. Additionally, 
photodamage is greatly minimized due to the use of lower energy photons and confinement of fluorescence emission to a diffraction-limited spot. ${ }^{8}$ This makes two-photon microscopy ideal for high-resolution ( $\mu \mathrm{m}$-scale), deep-tissue, real-time imaging and tracking of dynamic processes in cell biology. ${ }^{6}$

In order to realize deep-tissue optical imaging, it is crucial to develop tracers that not only exhibit the required photophysical properties (such as two-photon absorption and a fluorescence emission in the NIR), but also good biocompatibility, high sensitivity and specificity. Many organic dyes which show two-photon absorption are hydrophobic, leading to molecular aggregation in aqueous media and associated fluorescence quenching, which in turn limits their use as fluorescent probes for imaging.

The development of new types of fluorophores relying on aggregation-induced emission (AIE) has overcome the problem of aggregation-induced quenching. In particular, organic dyes that form J-aggregates have shown great potential for use in bio-imaging. J-aggregation refers to the selfassembly of molecules in a head-to-tail arrangement, resulting in delocalization of the $\pi$-electrons over the self-assembled molecular aggregate, which in turn leads to constructive coupling of the excited state transition dipoles. ${ }^{9,10}$ This results in several interesting photophysical characteristics, such as red-shifted absorption and emission spectra, narrow absorption and emission bands, high absorption coefficients and good quantum yields. ${ }^{9-11}$ Several organic dyes designed to form J-aggregates have been reported in the literature, such as perylene bisimide derivatives ${ }^{12}$, pyrrolopyrrole cyanine derivatives ${ }^{13}$, heptamethine cyanine dyes ${ }^{10,11,14}$ and porphyrin-based dyes $^{15-17}$. These chromophores are often complexed with polymers or encapsulated in nanoparticles (NPs) to impart stability and enhance their functionality. J-aggregates based on heptamethine cyanine dyes complexed with amphiphilic polymers to form stabilized polymeric micelles have 
been reported by Liu and coworkers. ${ }^{11,14}$ These polymer-stabilized J-aggregates have been further complexed with iron oxide nanoparticles ${ }^{11}$ and anticancer drugs $^{14}$ for photothermal and combination cancer therapy, respectively. Polymeric-micelle-stabilized J-aggregates of pyrrolopyrrole cyanine ${ }^{13}$ for in vivo imaging and squaraine ${ }^{5}$ for image guided photothermal therapy have also been reported.

Organic dyes exhibiting AIE have also been encapsulated in protein nanoparticles ${ }^{18,19}$ to enhance uptake by cancer cells and enable two-photon in vivo imaging. Silica-based NPs are also interesting options for the encapsulation of AIE based dyes, due to the availability of well-established synthesis protocols for controlling their size, their low intrinsic toxicity and ease of surface modification to increase their colloidal stability. ${ }^{20,21}$ In addition, the inert silica matrix can shield encapsulated dye molecules from the external environment, providing stability, optical transparency, and biocompatibility. ${ }^{21,22}$ Furthermore, the silicate matrix can be readily functionalized through silica surface chemistry, thereby providing an efficient strategy for the encapsulation of hydrophobic nanocrystals to obtain water-soluble nanomaterials. ${ }^{23-25}$ AIE based dyes have also been incorporated into silica NPs for bio-imaging. ${ }^{26-29}$

J-aggregates of a heptamethine dye have been prepared inside hollow mesoporous silica nanoparticles for NIR imaging by Sletten and coworkers. ${ }^{10}$ The silica nanoparticles have been shown to stabilize J-aggregates and facilitate high resolution in vivo imaging in mice. ${ }^{10}$ Prasad and coworkers have developed organically-modified silica (ORMOSIL) NPs loaded with a high concentration of AIE-based dyes for two-photon fluorescence imaging ${ }^{30}$ and photodynamic therapy when co-encapsulated with a photosensitizing drug. ${ }^{31}$

Encapsulation of organic crystal-state emitters in sol-gel matrices is an efficient strategy to produce bright nanotracers for bio-imaging applications. ${ }^{32-36}$ As described in our previous work, ${ }^{36}$ fluorescent organic@silicate core-shell nanoparticles (NPs) containing a crystalline organic core 
surrounded by a silicate shell, with a high loading of dyes (30-40 wt \%) are promising for bioimaging applications. These recently-described core-shell NPs, which comprise a nanocrystalline organic-dye core ( $c a 40-50 \mathrm{~nm}$ ) surrounded by a silicate shell, are prepared by a novel spray-drying technique which combines sol-gel chemistry and crystallization. ${ }^{32,36-38}$ The organic dyes used in the core-shell NPs are based on the D- $\pi$-A dipolar structure and are specifically designed to exhibit two-photon absorption in the crystal state. ${ }^{39}$ The permanent dipole moment associated with such structures results in strong dipole-dipole interactions, which in turn can induce specific organization and orientation of the molecules in the solid state. ${ }^{38}$ These core-shell NPs show fluorescence emission in the red, close to the biological transparency window, making them interesting candidates for deep-tissue imaging using two-photon microscopy.

In this study, we evaluate the potential of our novel organic@silicate core-shell NPs for two-photon in vivo fluorescence imaging. The CuAAC (Copper Catalyzed Azide to Alkyne Cycloaddition) click reaction ${ }^{40}$ was used to graft PEG chains onto the core-shell NPs to enhance their colloidal stability and "stealth" in blood by minimizing protein adsorption and opsonization. ${ }^{41-47}$ Classical DLS experiments coupled with small-volume DLS (confocal geometry) was used to characterize the colloidal stability of the NPs in different biological media with increasing complexity, starting with simulated body fluid (SBF) and various plasma proteins in increasing concentrations. Finally, we present, two-photon fluorescence properties in solution to demonstrate the potential of these NPs for two-photon fluorescence imaging both in vitro and in vivo.

\section{Experimental section}

Details of materials, reagents and characterization techniques are given in the supporting information

\section{Synthesis of core-shell NPs with clickable functions}


Figure 1 schematically summarizes the preparation of nanoparticles by spray-drying and the PEGylation step subsequently used to obtained stable colloidal suspensions.

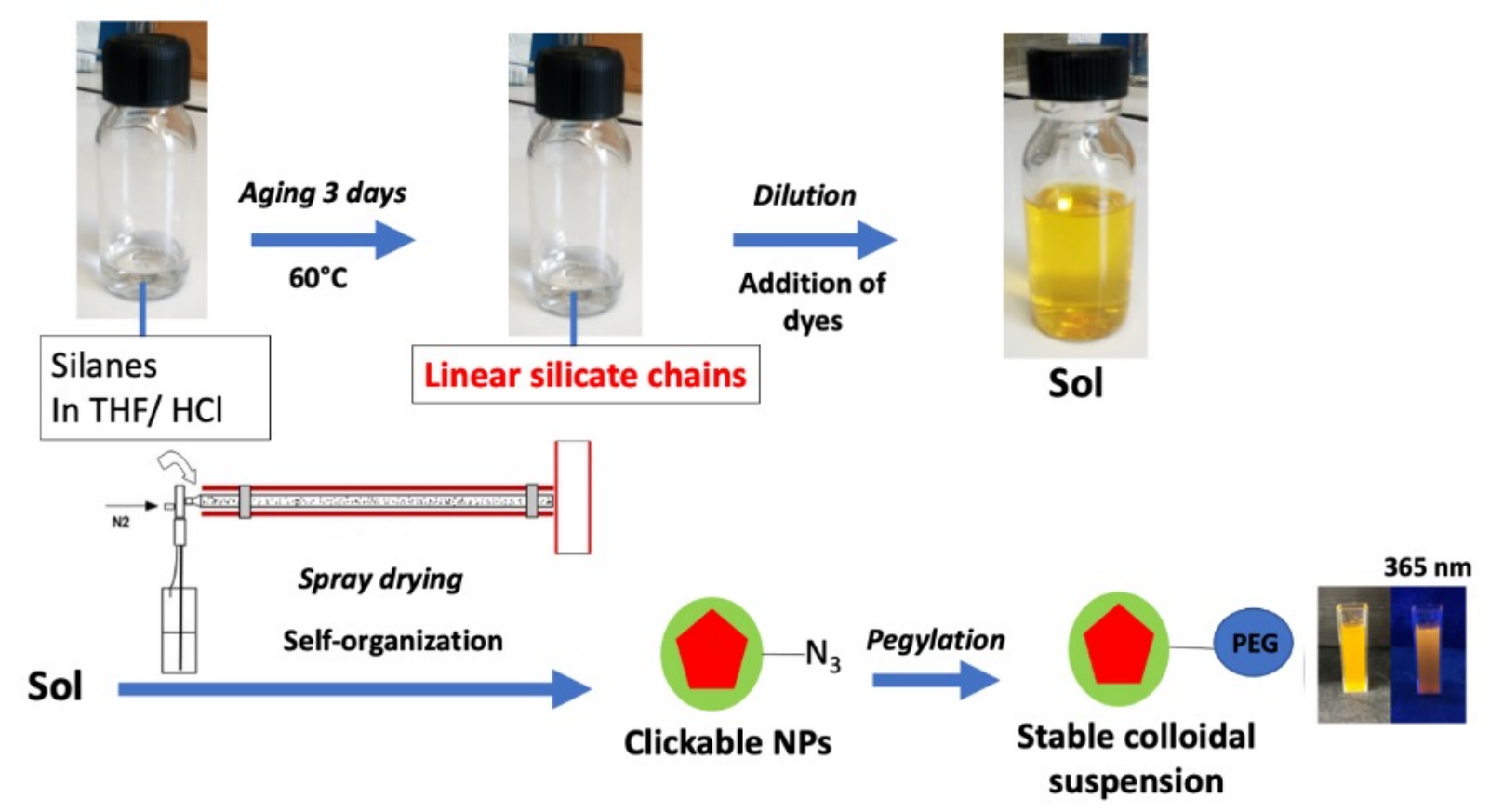

Figure 1 : Preparation of nanoparticles

The sols and the core-shell NPs (NP-I-N3) were prepared according to the published protocol ${ }^{36}$ using a 2:1 molar ratio of tetramethoxysilane (TMOS) and 1,2-bis(trimethoxysilyl)ethane (TMSE), with 3\% AzPTES with respect to Si and dilute aqueous $\mathrm{HCl}(1 \mathrm{M} \mathrm{HCl})$ in THF. Briefly, dilute $\mathrm{HCl}$ was added to the silicate precursors, previously dispersed in $5 \mathrm{~mL}$ of THF, under vigorous stirring, followed by aging for 3 days at $60^{\circ} \mathrm{C}$. Finally, additional THF was added after aging to reach a final volume of $60 \mathrm{~mL}$. The as-prepared sols were stored at $4{ }^{\circ} \mathrm{C}$. The dye was dissolved in the sol just before nebulization and spray-drying and the sol was nebulized under the conditions described in our previous work, yielding organic nanocrystals covered with a silicate layer. ${ }^{36}$ The crystalline nature of the organic core was ascertained by electron diffraction and by PXRD (Figure S1). A controlled partial dissolution of the silicate shell of the sprayed-dried NPs was performed by sonicating the NPs in water $(60 \mathrm{~mL})$ for 20 minutes in an ultrasonic bath $(150 \mathrm{~W})$, before increasing 
the solution $\mathrm{pH}$ to 12 by adding $1 \mathrm{M} \mathrm{NaOH}$. The resulting suspension was stirred for $24 \mathrm{~h}$ in a closed bottle. The NP suspension was then subjected to centrifugation for 5 minutes at $5000 \mathrm{rpm}$ and the supernatant was filtered using $5 \mu \mathrm{m}, 1.2 \mu \mathrm{m}$ and $0.8 \mu \mathrm{m}$ syringe filters, to remove large aggregates. This step was followed by neutralization of the NP suspension to $\mathrm{pH} 7.4$ by the dropwise addition of $1 \mathrm{M} \mathrm{HCl}$. The resulting NP suspensions were used for fluorescence spectroscopy measurements. The $\mathrm{pH}$ of the neutralized core-shell NPs for click functionalization was further stabilized using $0.133 \mathrm{M}$ phosphate buffer by adding a volume corresponding to $10 \%$ of the volume of the NP suspension.

\section{CUAAC functionalization of core-shell NPs}

The treated core-shell NPs were functionalized using the CuAAC click reaction, following the protocol described by Moitra et al. $^{48}$ Five equivalents of propargyl-PEG 5000-OMe for each equivalent of AzPTES were first weighed into a bottle in which the treated NP suspension was added under constant stirring. One equivalent each of tris-(3-hydroxypropyltriazolylmethyl)amine (THPTA) and of the pre-catalyst, copper sulphate $\left(\mathrm{CuSO}_{4} .5 \mathrm{H}_{2} \mathrm{O}\right)$, were then added to this solution. Argon was bubbled into the colloidal suspension for a few minutes to remove any traces of oxygen before the addition of four equivalents of sodium ascorbate. More Ar was bubbled in the suspension and the bottle was then closed and kept under constant stirring at room temperature for 24 hours. Purification of the functionalized NPs (NP-I-PEG) was performed by dialyzing the NP suspension (10 mL in Pur-A-Lyzer tubes with 3.5 kDa pore size) against 2.5 L of 0.0133 M PB for two days. The purified NP suspensions were concentrated using Vivaspin 2 (2 mL) concentrators. Centrifugation was performed at $3000 \mathrm{rpm}$ for 3 minutes on volumes of $2 \mathrm{~mL}$. Successive centrifugations were performed to increase the concentration of particles in suspension.

\section{Steady-state spectroscopic experiments under resonant one-photon absorption conditions}


The two-photon excitation cross-section measurements were performed by detecting the twophoton excitation-induced fluorescence (TPEF) of the NPs compared to the signal of a $\mathrm{pH} 13$ aqueous solution of fluorescein $\left(\mathrm{c}=10^{-5} \mathrm{~mol} / \mathrm{L}\right)$ used as a standard. ${ }^{49,50}$ The experimental setup is based on a femtosecond Ti:Sapphire laser (Chameleon Ultra I, Coherent, $80 \mathrm{MHz}$ pulse repetition rate, 140 fs pulse duration) allowing for tunable excitation between 700 and $1040 \mathrm{~nm}$. The laser intensity was adjusted by using a continuously variable neutral density filter to ensure constant excitation power in the tuning range. The laser power was monitored by a NIST traceable thermal detector (S405C, Thorlabs). The laser beam was focused into the $10 \times 10 \mathrm{~mm}$ quartz (QZS) sample cell by a $100 \mathrm{~mm}$ focal length lens. The fluorescence signal was detected at $90^{\circ}$ to the excitation beam. The cell position was adjusted so that the optical path of both the excitation beam and the fluorescence emission was minimized in the cell, thus reducing experimental artefacts such as inner-filter effects, scattering and solvent absorption. The emitted fluorescence was filtered using a low-pass interference filter and delivered to a fiber optic spectrometer (AvaSpec-EVO, Avantes).

\section{In vitro and in vivo assays}

For in vitro experiments, MCF-7 human breast cancer cells were used (purchased from ATCC) and cultured in Dulbecco's modified Eagle’s medium (DMEM) supplemented with $10 \%$ fetal bovine serum and $50 \mu \mathrm{g} \mathrm{mL} \mathrm{mL}^{-1}$ gentamycin and maintained under a humidified atmosphere at $37^{\circ} \mathrm{C}$ containing 5\% $\mathrm{CO}_{2}$ (incubator). Cytotoxic studies were realized by seeding MCF-7 on 96-well plates (2000 cells/well). The day after seeding, cells were incubated with increasing concentrations of NP-I-PEG (from 0.05 to $50 \mu \mathrm{g} \mathrm{mL}^{-1}$ ). One day after, the plates intended for "light cytotoxicity" study were exposed during $4 \mathrm{~h}$ at the sunlight, then they were replaced in the incubator in the dark for the remaining $48 \mathrm{~h}$. The plates “dark cytotoxicity” stayed in the incubator $72 \mathrm{~h}$ without interruption. At the end of the 3 days, a MTT assay was performed to determine the cell viability. Briefly, cells were incubated for $4 \mathrm{~h}$ with $0.5 \mathrm{mg} \mathrm{mL}^{-1}$ of MTT (3-(4,5- dimethylthiazol-2-yl)-2,5- 
diphenyltetrazolium bromide; Promega) in culture medium. The MTT/medium solution was then removed and the precipitated crystals were dissolved in EtOH/ DMSO (v/v). The solution absorbance was read at $540 \mathrm{~nm}$ in a microplate reader. All values are reported in relation to the control values (without any treatment) which are considered as $100 \%$ living cells.

For fluorescence imaging on living cells, MCF-7 cells were seeded in glass bottom dishes (World Precision Instrument, Stevenage, UK), one day prior to commencing the experiment, at a density of $10^{6}$ cells. $\mathrm{cm}^{-2}$. Cells were then washed once and incubated in $1 \mathrm{~mL}$ culture medium with or without NP-I-PEG at a concentration of 10 or $25 \mu \mathrm{g} \mathrm{mL}^{-1}$ for $24 \mathrm{~h}$. Two hours before the end of the incubation, cells were loaded with LysoTracker green $(1 \mu \mathrm{M}$, Molecular Probes, Life technologies, Oregon, USA); 15 minutes before the end of incubation, cells were loaded with Hoechst $33342\left(10 \mu \mathrm{g} \mathrm{m}^{-1}\right.$, Invitrogen, Cergy Pontoise, France) for nuclear staining, or with green CellMask (5 $\mu \mathrm{g} \mathrm{mL}^{-1}$, Invitrogen, Cergy Pontoise, France) for membrane staining. Before microscope observation, cells were gently washed with cell culture medium and scanned with a LSM 780 LIVE confocal microscope (Carl Zeiss, Le Pecq, France), at $450 \mathrm{~nm}$ (continuous) or 900 nm using the Chameleon (pulsed) laser for NP-I-PEG visualization. Samples were excited at 561 $\mathrm{nm}$ for membranes, at $760 \mathrm{~nm}$ for nuclei and at $488 \mathrm{~nm}$ for lysosomes.

All images were performed with a high magnification (63×/1.4 OIL DIC Plan-Apo).

For mice studies, all experimental procedures were carried out in accordance with European regulations for animal use and were authorized by the local Ethics Committees (CEEA-55/CETEA2015-0038) and the French Ministry of National Education and Research (APAFIS\# DR2017-58). For two-photon in vivo imaging, 8-week-old CX3CR $1^{\mathrm{eGFP} /+}$ mice, expressing GFP only in microglia in the central nervous system (SNC), were used. For transcranial imaging, a thinnedskull cortical window preparation was performed over the somatosensory cortex, where the skull 
was thinned to 20-30 $\mu \mathrm{m}$. Mice were deeply anesthetized with isoflurane (3-4\%, Isovet, Piramal Healthcare, UK Ltd.) and mounted in a stereotaxic apparatus (D. Kopf Instruments). Carprofen (5 $\mathrm{mg} / \mathrm{kg}$ s.c.) was injected at the beginning of the surgery to reduce post-surgical pain and inflammation. After the skull was exposed, a thin custom-made metal implant was glued allowing the area over the somatosensory cortex to be delimited. The skull was then carefully thinned using a high-speed dental drill. To avoid heat-induced damage, drilling was repeatedly interrupted and cold sterile saline was applied. When a 20 to $30 \mu \mathrm{m}$ skull thickness was reached, a thin layer of cyanoacrylate glue was applied and a cover glass was placed on top of the thinned skull.

For in vivo imaging sessions, mice were anesthetized with a mixture of ketamine $(100 \mathrm{mg} / \mathrm{kg})$ and xylazine $(10 \mathrm{mg} / \mathrm{kg})$. Their body temperature was maintained at $37^{\circ} \mathrm{C}$ using a heating pad. A retroorbital injection of $200 \mu \mathrm{L}$ of a NP solution at a concentration of $80 \mu \mathrm{g} / \mathrm{mL}$ was performed while the mouse was deeply anesthetized. Two-photon microscopy observations were performed on a Bruker Ultima microscope (Insight 3X laser, Spectra Physics), equipped with a 20× waterimmersion objective (0.95 N.A. Olympus). Green (CX3CR1 ${ }^{\mathrm{eGFP} /+}$ cells) and red (NPs) fluorescence were separated by a $560 \mathrm{~nm}$ dichroic mirror coupled to 525/50 nm and 650/40 emission filters. Images were acquired every 0.9 seconds over an area of $200 \times 200 \mu \mathrm{m}^{2}$ and a resolution of $521 \times 521$ pixels, and laser power was maintained below $30 \mathrm{~mW}$. The open source software packages ImageJ and Icy, created by BioImage Analysis Lab, Institut Pasteur (France; http://icy.bioimageanalysis.org/) were used for image treatment, while drift correction was applied using custom-written Matlab software to correct image drift during acquisition.

\section{Results and discussion}


Due to the presence of organic nanocrystals at the core of the NPs, surface functionalization had to be performed in aqueous solution to minimize dissolution of the hydrophobic core. Our previous protocol $^{36}$ was thus adapted to incorporate an azide functionality within the NPs during sol-gel synthesis (figure 1), ${ }^{48,51}$ for subsequent functionalization with alkyne-modified moieties via the CuAAC reaction in pure water. In addition to TMOS (2 eq.) and TMSE (1 eq.), 3 mol\% of (3-azidopropyl)triethoxysilane (AzPTES) was added to the starting sol. Using dye I, the morphology and size distribution of the NPs (NP-I-N 3 ) obtained (FESEM image, Figure 2) were unaltered after the introduction of this new silicate precursor into the sol (Figure S2).

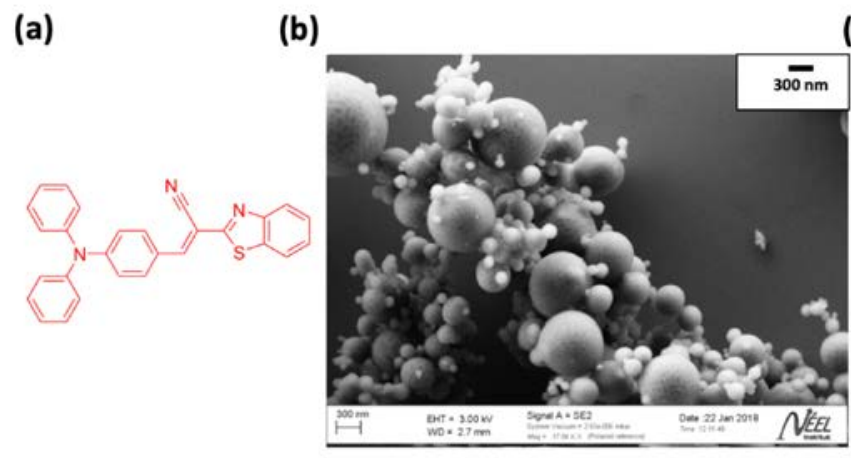

(c)

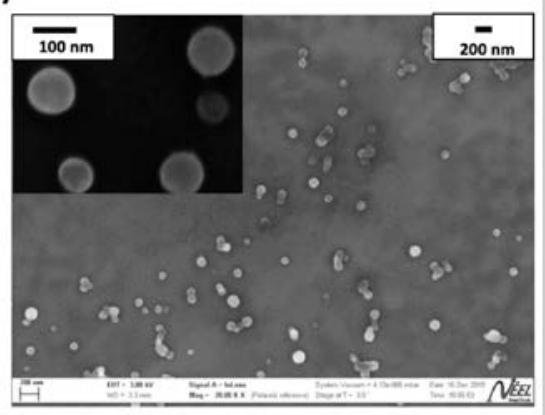

Figure 2 : (a) Structure of the dye (E)-2-(benzo[d] thiazol-2-yl)-3-(4-diphenylamino)phenyl)acrylonitrile . (b) FESEM imaging of as-synthesized NP-I-N $\boldsymbol{N}_{3}$ (C) NP-I-PEG after size selection and PEGylation (right).

Fourier transform infrared spectroscopy (FTIR) confirmed the presence of a high loading of dye (30-40 wt $\%$ as previously determined by TGA ${ }^{36}$ ) and the azide groups within the NPs (supporting information, Figure S3). Indeed, the bands corresponding to $-\mathrm{CN}\left(2210 \mathrm{~cm}^{-1}\right)$ and $\mathrm{C}=\mathrm{C}\left(1573 \mathrm{~cm}^{-1}\right)$ from the dye are clearly visible in the spectrum of NP-I-N3, and absent in dye-free NPs. The integrity of the encapsulated dye molecule after synthesis was demonstrated by absorption and fluorescence spectroscopy after dissolving the NP-I-N3 NPs in ethanol.

To disperse the dry NP powder obtained by spray-drying, a partial dissolution of the silicate shell with sodium hydroxide at $\mathrm{pH} 12$ was performed for $24 \mathrm{~h}$ in order to separate the chemically aggregated NPs and impart a strongly negative $\zeta$ potential. After this treatment, the suspension was 
centrifuged to sediment the largest particles and aggregates, then the supernatant containing the desired NPs was filtered using $5 \mu \mathrm{m}$ to $0.8 \mu \mathrm{m}$ cellulose acetate membranes and finally neutralized to $\mathrm{pH} 7.5$.

The suspension of NPs as prepared in pure water was characterized by DLS measurements. The auto-correlation functions of the measured intensities were derived and fitted to determine the average size of the NPs and the width of their size distribution. The latter is shown in Figure S4-a. in the supporting information, as a function of the number of NPs and as a function of their scattered intensities; the latter favors the largest NPs, since scattering intensity increases with increasing particle size. These suspensions were stable for several hours, although the subsequent addition of sodium chloride up to $0.9 \mathrm{wt} \%$, which corresponds to blood salinity, had a strong negative impact on the colloidal stability. This effect was monitored by DLS measurements, performed every minute over two hours, that showed a progressive increase of the average size of the scattering species as a function of time (SI, Figure S4-b). This reflects the salt-induced aggregation of NPs, which was further confirmed by observing the NP sedimentation on a longer time scale, namely during fluorescence spectroscopy experiments performed over 5 hours (SI, Figure S4-b). This colloidal instability in saline environments makes it necessary to functionalize the surface of the NPs.

Accordingly, we covalently grafted polyethylene glycol $(\mathrm{Mn}=5000)$ onto the organosilica surface of the core-shell NPs using the CuAAC click reaction of NP-I-N 3 with propargyl-PEG-OMe (Figure 3). 


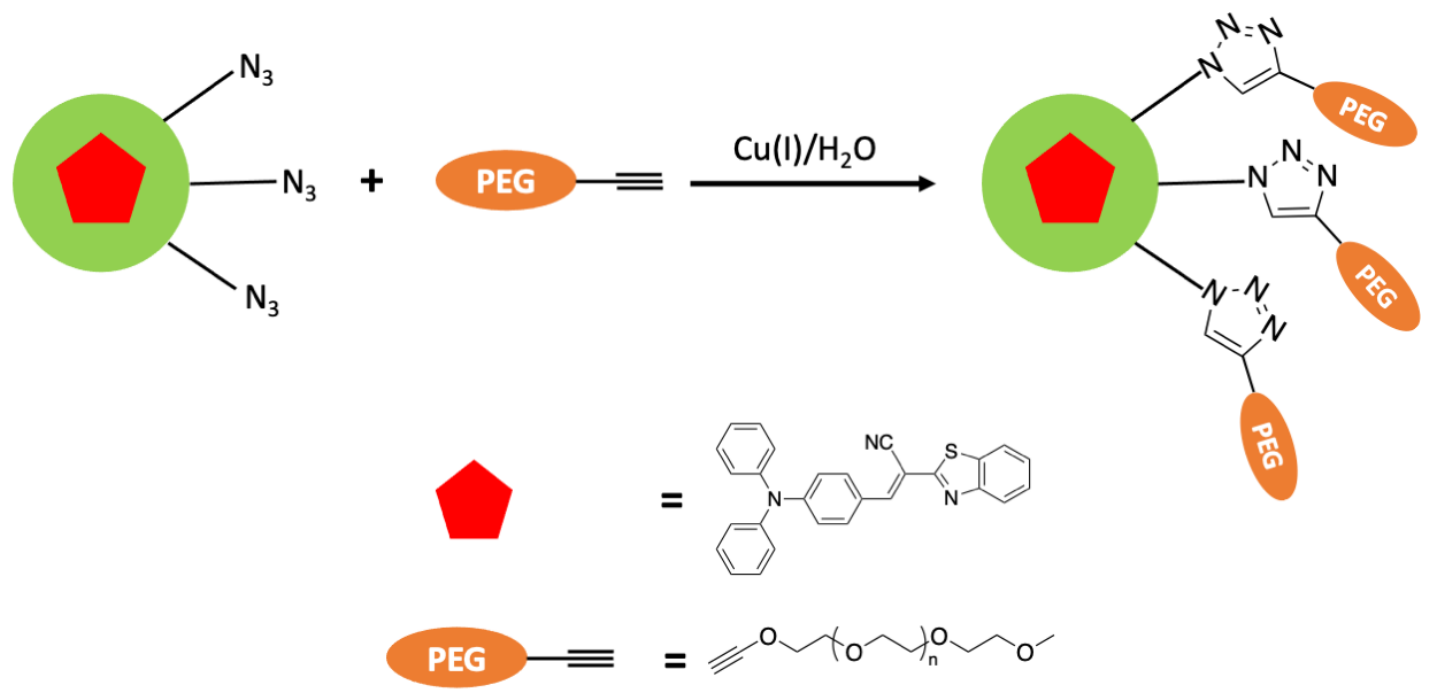

Figure 3 : CuAAC click functionalization of NPs

The comparison of the FTIR spectra before and after functionalisation (SI - Figure S3) demonstrates the success of the CuAAC reaction, with a strong decrease in the azide band ( $N=\mathrm{N}=\mathrm{N}$ stretch) intensity at $2100 \mathrm{~cm}^{-1}$ and the appearance of bands corresponding to the $\mathrm{C}-\mathrm{H}$ bonds of the PEG chains in the range $2800-2900 \mathrm{~cm}^{-1}$. The efficiency of PEG grafting is also shown by the decrease in $\zeta$ potential at $\mathrm{pH} 7.4$ from $-40 \mathrm{mV}$ before functionalization to around $-10 \mathrm{mV}$ after pegylation.

After centrifugation, the NP-I-PEG colloidal suspensions $(80 \mu \mathrm{g} / \mathrm{mL})$ in pure water were again characterized by DLS measurements. The analyses of the auto-correlation functions lead to an intensity-weighed size distribution centred at $c a 170 \mathrm{~nm}$ (figure S 6 (a)) and a width corresponding to sizes ranging from 100 to $300 \mathrm{~nm}$, in agreement with the SEM micrographs showing NPs of about $100 \mathrm{~nm}$ and small aggregates (Figure 2).

To further characterize the suspensions, DLS and FCS experiments under a confocal microscope were also performed. ${ }^{52}$ The experiments involve measuring the elastic (for DLS) or inelastic scattering (here fluorescence for FCS) of the NPs as their Brownian motion brings them to diffuse through a small observation volume where they are illuminated and scatter light. The measured 
intensities exhibit temporal fluctuations related to an increase (or decrease) of signal each time a NP enters (or leaves) the confocal volume. Consequently, the amplitudes and duration of these fluctuations are related to the mean number, $\langle N\rangle$, of NPs, and to their residence time, $\tau_{D}$, respectively, within the observation volume. Both parameters can be obtained by adjusting their values through fitting of the intensity auto-correlation function, $A C F=G(t)-1$, where $G(t)=$ $\frac{\langle I(\tau) I(t+\tau)\rangle}{\langle I(\tau)\rangle^{2}}$. The size and the concentration of the NPs are further obtained from the experimental determination of $\langle N\rangle$ and $\tau_{D}$. The detailed procedure is given in supporting information.

A series of ten DLS experiments were performed with this setup, leading to a slightly different ACF for each measurement due to the dispersion of the NPs and to the fact that only a few of them are measured simultaneously, in contrast to the conventional DLS equipment. An averaged ACF is presented in Figure 4-a together with the calculated one using $G(0)=21.8$ and $\tau_{D}=3 \mathrm{~ms}$. Knowing the confocal volume $V_{c}=0.7 \mu \mathrm{m}^{3}$ and its radius $r_{0}=190 \pm 30 \mathrm{~nm}$ from calibration measurements, we obtain a mean concentration $C=2.1 \times 10^{10} \mathrm{p} / \mathrm{mL}$ and a mean hydrodynamic diameter $d_{H}=160 \pm 30 \mathrm{~nm}$. The different ACFs present characteristic residence times ranging from 2.5 to $4 \mathrm{~ms}$, which correspond to hydrodynamic diameters ranging from 80 to $300 \mathrm{~nm}$, and $\langle N\rangle$ value ranging from 0.012 to 0.024 , corresponding to a concentration $C=2.5 \pm 0.8 \times 10^{10}$ $\mathrm{p} / \mathrm{mL}$. These results are in full agreement with the NP sizes obtained from the commercial DLS, and with the initial concentration of $80 \mu \mathrm{g} / \mathrm{mL}$ corresponding to a concentration of $2 \times 10^{10} \mathrm{p} / \mathrm{mL}$ when considering NPs of $170 \mathrm{~nm}$ in diameter and of density of 1.5 . 


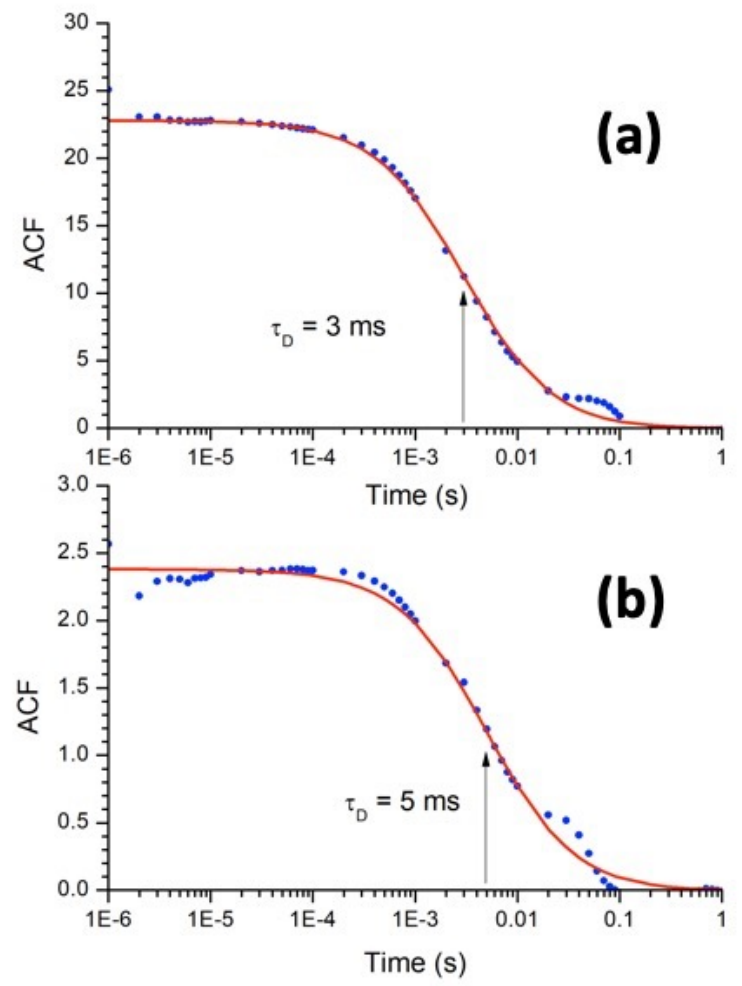

Figure 4: (a) DLS experimental ACF averaged over ten measurements (dots) and calculated fit (line) with G(0)= 21.8 and $\tau_{D}=3 \mathrm{~ms}$ and (b) FCS experimental ACF (dots) averaged over ten measurements and calculated one (line) with $G(0)=2.4$ and $\tau_{D}=5 \mathrm{~ms}$.

FCS measurements were analysed using exactly the same experimental procedure. As can be seen in Figure 4-b, the value $G(0)=2.4$ is significantly smaller than that of the DLS ACFs due to a much weaker signal-to-noise ratio. However, taking the latter into account $\left(c^{2} \sim 0.055\right.$ and $\langle N\rangle=c^{2} / G(0)$, see SI), a concentration $C=3.3 \times 10^{10} \mathrm{p} / \mathrm{mL}$ is obtained, which is in accordance with the concentration measured in DLS. This similarity indicates that all the NPs emit a fluorescence signal, hence confirming the presence of the fluorescent crystal within the core/shell NPs. The characteristic residence time, $\tau_{D}=5 \mathrm{~ms}$, is-larger than the values obtained from DLS. This is attributed to the weakness of the fluorescence signal that favours the detection of the largest NPs and to a possible retardation of the NPs due to weak optical trapping within the confocal volume resulting from the much larger incident power used as compared to that in DLS. ${ }^{53}$ 
Blood is a complex medium containing mainly high amounts of salts (ionic strength around 0.15 M), proteins and cells. To predict the colloidal stability in blood, we undertook a series of DLS analyses in environments of increasing chemical complexity, approaching step by step the plasma composition.

The first step was to check the stability of the colloidal solution of PEGylated NPs (NP-I-PEG) in the presence of a salty medium and in the presence an ionic environment simulating that of blood at $37^{\circ} \mathrm{C}$. DLS measurements were performed in solutions of the functionalized NP-I-PEG in pure water and at $25^{\circ} \mathrm{C}$ and were compared to those obtained at $37^{\circ} \mathrm{C}$ in pure water, with $0.9 \mathrm{wt} \% \mathrm{NaCl}$, for the salty medium, and with simulated body fluid (SBF) which mimics blood's ionic composition (Table S1). The size distributions of the NPs obtained from these DLS measurements are presented in supporting information (Figure S6). Neither the temperature, the salty medium nor the SBF influences the size distribution of the NPs, which are similar to that of the NP-I-PEG in pure water and at $25^{\circ} \mathrm{C}$. Moreover, in all situations, the functionalized NP-I-PEG were stable with a mean size lower than $200 \mathrm{~nm}$, in contrast to the unfunctionalized $\mathbf{N P}-\mathbf{I}-\mathbf{N}_{\mathbf{3}}$.

The colloidal stability of the NPs was then tested in the presence of plasma proteins at $37^{\circ} \mathrm{C}$, at least 15 minutes after addition. The latter constitute a major fraction of blood, with serum albumins constituting $55 \%$ of the total amount of blood plasma proteins, globulins constituting $38 \%$ and the remaining $7 \%$ constituted mainly by fibrinogen. It is now well documented that the interaction of NPs with those proteins lead to the formation of a protein corona ${ }^{54}$, which may lead to aggregation or recognition by the macrophages. It is therefore essential to check the colloidal stability and possible aggregation of NP-I-PEG in the presence of plasma proteins. Incremental experiments were performed: (i) the interaction of the NPs with bovine serum albumin (BSA) as a model albumin was first probed; (ii) the effect of the second most abundant protein in the blood plasma, 
namely $\gamma$-globulin, was then investigated; and finally, (iii) the colloidal stability of the functionalized NPs was investigated in an environment composed of a combination of the individually tested proteins (SBF, BSA and $\gamma$-globulin). As previously, the colloidal stability was investigated by DLS measurement performed at $37^{\circ} \mathrm{C}$. During the first step, an increasing amount of BSA in SBF, ranging from 6 to $60 \mathrm{mg} / \mathrm{mL}$, was added to the NP-I-PEG suspensions. The limiting concentration of $60 \mathrm{mg} / \mathrm{mL}$ corresponds to the maximum concentration encountered in blood. DLS measurements were also performed on a pure BSA solution as a reference. Figure 5-a presents the normalized ACFs of the BSA and of the NPs in SBF together with their fit calculated for characteristic time decays of $18 \mu$ s for BSA and $910 \mu$ s that corresponds to hydrodynamic diameters of $8.4 \mathrm{~nm}$ and $200 \mathrm{~nm}$, respectively (which are the expected values). As the characteristic residence time of the BSA and that of the NPs are very different, their respective contribution to the ACF are not correlated and therefore simply sum to yield the final ACF. At short times, the time decay due to the BSA becomes more visible as its concentration increases. At longer time, and at low concentrations in BSA (6 and $15 \mathrm{mg} / \mathrm{mL}$ ), the ACFs are very similar to that of the NPs only in the presence of SBF, indicating the stability of the colloidal solutions. At higher BSA concentrations (30 and $60 \mathrm{mg} / \mathrm{mL}$ ), a slight shift of the ACFs is observed at longer times, consistent with an increase in the polydispersity of the solutions. To obtain more quantitative results, the ACFs were fitted and the NPs size distributions were obtained. The latter are displayed in Figures 5-b,c, both as number-and intensity-weighted distributions. At low concentrations of BSA (6-15 $\mathrm{mg} / \mathrm{mL}$ ), the size distributions are very similar to those obtained for the NPs in pure water, confirming that no aggregation occurred. At higher concentrations, a peak at $8 \mathrm{~nm}$ appears in the number-weighted distribution, while that of the NPs disappears. This corresponds to the observation of the diffusion of the albumin molecules whose concentration becomes important. 
The situation is different in the case of the intensity-weighted distributions, as the latter favors the larger objects due to their larger contribution to the observed signal. A slight aggregation was observed only at the highest concentration of BSA $(60 \mathrm{mg} / \mathrm{mL})$, resulting in a size increase from $220 \mathrm{~nm}$ to $~ 260 \mathrm{~nm}$, while only a slight broadening of the distribution is observed for the $30 \mathrm{mg} / \mathrm{mL}$ concentration in BSA. However, the observation of aggregation at a BSA concentration of $60 \mathrm{mg} / \mathrm{mL}$ is not problematic, as this concentration is higher than the upper limit of the normal albumin concentration in the body. The stability of the functionalized NPs suspension under nominal conditions is therefore confirmed.
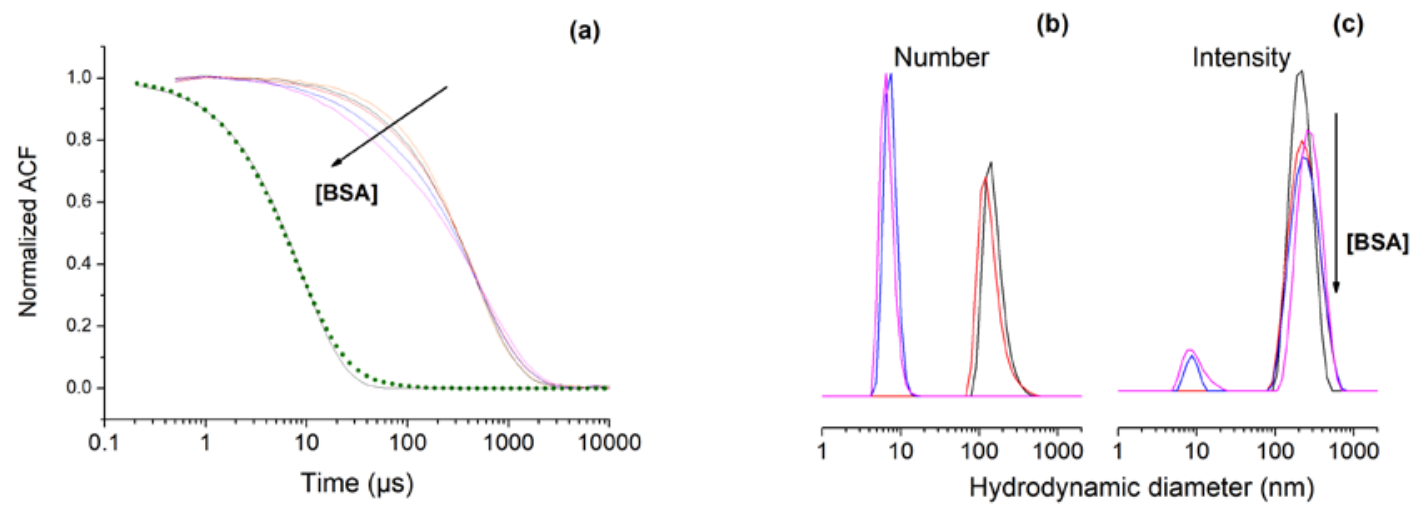

Figure 5 : (a) Normalized autocorrelation data corresponding to NP-I-PEG in SBF with BSA at a concentration of $0 \mathrm{mg} / \mathrm{mL}$ (orange), $6 \mathrm{mg} / \mathrm{mL}$ (black), $15 \mathrm{mg} / \mathrm{mL}$ (red), $30 \mathrm{mg} / \mathrm{mL}$ (blue) and $60 \mathrm{mg} / \mathrm{mL}$ (pink); of pure BSA in PBS (green). Fits of the green and orange plots (black) give a characteristic time of $18 \mathrm{~ms}$ for BSA and $910 \mathrm{~ms}$ for the NP-I-PEG. (b) Number and (c) Intensity distributions of NP-I-PEG in SBF at $37^{\circ} \mathrm{C}$ with the different concentrations of BSA.

The effect of the second most abundant protein in blood plasma, $\gamma$-globulins, which are constituents of the immune system, was investigated next. DLS measurements were performed on a solution containing only $\gamma$-globulin molecules and on solutions of NPs in SBF with $\gamma$-globulin at a concentration of 10, 25 and $45 \mathrm{mg} / \mathrm{mL}$. The measured ACFs are displayed in SI (Figure S7-a), together with that of the NPs in SBF. The contribution of the $\gamma$-globulin in ACFs appears as a time decay of $52 \mu$ s (Figure S7-a), which corresponds to a diameter of $11.5 \mathrm{~nm}$ for the equivalent spherical molecule. As for BSA, this scattering is expected to contribute to that of the NPs when 
$\gamma$-globulin is added to the solution without adsorbing on the NPs. This is in fact what is observed on the ACFs (Figure S7-b), which present a time decay at short times whose intensity increases with increasing concentration of $\gamma$-globulin. However, no clear changes are observed in the ACFs at longer times, indicating the total absence of aggregation. These results were confirmed by the size distribution obtained from the ACF fits: the number distribution shows no contribution of the NPs even at the lowest concentrations (Figure S7-c), but exhibits a peak at 10-12 nm corresponding to the size of the $\gamma$-globulin. On the other hand, in the intensity distribution (Figure S7-c), the NP contribution was clearly visible in all cases due to the higher contribution of the larger size to the measured signal. The intensity distributions show that the NPs were stable at the different concentrations of $\gamma$-globulins (10-45 mg/mL), with a size distribution peaking at less than $200 \mathrm{~nm}$, thus confirming the colloidal stability of the NP in the presence of $\gamma$-globulin.

Finally, the colloidal stability of the functionalized NPs was investigated in an environment composed of a combination of individually tested proteins at their highest concentrations, ie in SBF with $60 \mathrm{mg} / \mathrm{mL}$ of BSA and $45 \mathrm{mg} / \mathrm{mL}$ of $\gamma$-globulin. A small quantity $(1 \mathrm{mg} / \mathrm{mL})$ of glucose was also added to better simulate the plasma composition. Figure S8 presents the measured ACF together with the calculated fit. The latter was obtained by least square fitting and considering two independent exponential functions: one for the contribution of the small molecules and one for the contribution of the NPs. The derived characteristic time for the NPs is $\tau=1140 \mu$ s, which corresponds to a mean hydrodynamic diameter of $250 \mathrm{~nm}$. This is in agreement with the previous observation of a slight aggregation upon addition of $60 \mathrm{mg} / \mathrm{mL}$ albumin to the solution. This result is promising as it indicates that no further aggregation appears under these drastic conditions.

The previous measurements highlight a particularly good colloidal stability, with only minor aggregation of the NPs observed upon adding BSA at $60 \mathrm{mg} / \mathrm{mL}$. However, since standard DLS 
measurements are carried out in a large observation volume of several $\mathrm{mm}^{3}$, many NPs are illuminated at the same time and the measured size distributions average the different sizes of the NPs. Therefore, possible NP size heterogeneities that appear in the early stages of the aggregation process could be missed. On the contrary, when performing DLS experiments in a small observation volume, only a few tens of NPs diffuse during the whole acquisition time of a few seconds and the presence of a unique aggregate that scatters much more light than the small NPs is sufficient to modify the entire ACF. The former can therefore be detected even at very low concentrations.

To support the results obtained from the standard DLS measurements, DLS studies under our confocal microscope were therefore undertaken on the NP solutions containing SBF and an increasing concentration of BSA, ranging from 6 to $60 \mathrm{mg} / \mathrm{mL}$. As before, ten ACFs were measured for each BSA concentration and the mean ACFs are shown in Figure 6. The latter were normalized to ease the comparison of the characteristic times. At 6 and $15 \mathrm{mg} / \mathrm{mL}$ of BSA the ACFs present a mean characteristic time $\tau_{D}=3.2 \mathrm{~ms}$ and $\tau_{D}=3.5 \mathrm{~ms}$, respectively, slightly higher than the mean characteristic time, $\tau_{D}=3 \mathrm{~ms}$, of the NPs with no BSA, but within the range of 2.5-4 ms due to the natural size dispersion of the NPs. At 30 and $60 \mathrm{mg} / \mathrm{mL}$ of BSA, the ACFs exhibit a mean characteristic time $\tau_{D}=4.2 \mathrm{~ms}$ and $\tau_{D}=5.5 \mathrm{~ms}$, respectively, which are higher than the value for the non-aggregated NPs and increase with increasing concentration of BSA. These first results confirm the previous observation of the absence of aggregation for 6 and $15 \mathrm{mg} / \mathrm{mL}$ of BSA, the broadening of the size distribution at $15 \mathrm{mg} / \mathrm{mL}$ corresponding to a slightly larger mean characteristic time; and the start of an aggregation process at $60 \mathrm{mg} / \mathrm{mL}$ of BSA. The ten different ACFs performed for each concentration were also analyzed individually. For each concentration, the measured ACFs were separated into two categories: those with a characteristic time within the range 2.5-4 ms for the individual NPs and those with higher characteristic time. The mean ACF of 
each category in displayed in Figure S10, for the four concentrations in BSA and in Figure 6-b for the $60 \mathrm{mg} / \mathrm{mL}$ concentration. Up to $30 \mathrm{mg} / \mathrm{mL}$, the majority of the ten measured ACFs exhibit characteristic times consistent with the diffusion of individual NPs with mean diffusion times $\tau_{D}=$ 2.8 - $3.2 \mathrm{~ms}$, corresponding to the expected hydrodynamic diameter $d_{H}=150-180 \mathrm{~nm}$. For these three concentrations, only one or two ACFs out of ten exhibit a higher characteristic time corresponding to the diffusion of small aggregates typically composed of 2-5 NPs. ${ }^{52,55}$ At a BSA concentration of $60 \mathrm{mg} / \mathrm{mL}$, we observe a larger number of ACFs exhibit characteristic times which are not consistent with the diffusion of individual NPs, as their value range from $\tau_{D}=4.5$ to $11 \mathrm{~ms}$. This indicates that more and larger aggregates are formed at this concentration as compared to the lower concentrations, thereby confirming the beginning of an aggregation process.

Finally, the concentration of the individual NPs within the four different solutions was estimated from the ACFs with no aggregates. The obtained mean and individual values are displayed in Figure 6-c for each concentration in BSA as well as for the NPs solution with no BSA. The blue rectangle indicates the width of the error bar on the estimation of the initial NP concentration. One can observe that the concentration of the individual NPs is stable at 6 to 15 $\mathrm{mg} / \mathrm{mL}$ in BSA and decreases slightly at $30 \mathrm{mg} / \mathrm{mL}$ (although the measured value is still the same within the error bars), excluding the coagulation of the NPs. On the contrary, an actual decrease in the concentration of individual NPs is observed at $60 \mathrm{mg} / \mathrm{mL}$ in BSA, which again is consistent with an aggregation process. However, even in this latter case, almost half of the initial individual NPs are still present as isolated NPs in the solution. 

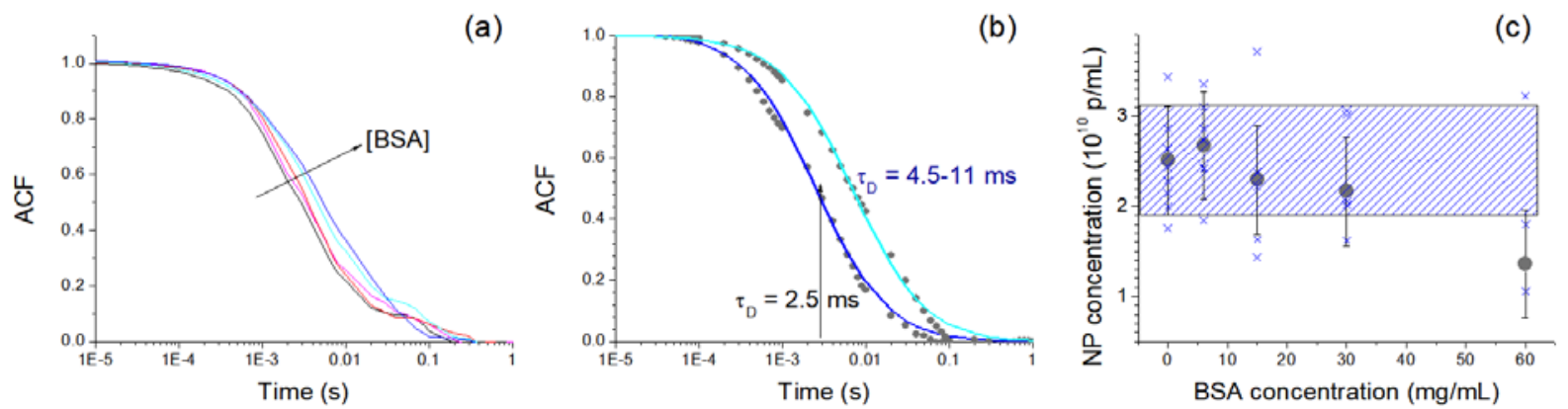

Figure 6: (a) Mean ACFs normalized to one measure of the NPs solutions with 0 (black line), 6 (light red), 15 (dark red), 30 (light blue) and $60 \mathrm{mg} / \mathrm{mL}$ of BSA (dark blue). (b) at $60 \mathrm{mg} / \mathrm{mL}$ of BSA, the average ACF is the sum of two of ACFs: one corresponding to the diffusion of isolated NPs with a mean characteristic time of $2.5 \mathrm{~ms}$ (dark blue) and one corresponding to the diffusion of small aggregates with a mean characteristic time of $7.5 \mathrm{~ms}$ and values ranging from 4.5 to $11 \mathrm{~ms}$ depending on the different ACFs. (c) Mean concentration of the isolated NPs (gray dots) and concentration extracted from the different ACFs (Blues crosses) as a function of the BSA concentration.

Figure 7 shows the resonant one-photon absorbance spectrum of the aqueous solution of the NP-I-PEG superimposed with their two-photon excitation spectrum, and its one-and two-photon excited fluorescence spectra. The linear absorbance spectrum exhibits a maximum at $464 \mathrm{~nm}$. As shown earlier, ${ }^{39}$ this large absorbance is associated with a charge transfer transition characteristic of donor-acceptor type structures. The absorbance spectrum is further broadened by light scattering due to the relatively large particles giving a non-negligible contribution even above $600 \mathrm{~nm}$. The two-photon absorbance spectrum (Figure $7-a)$ peaks at $930 \mathrm{~nm}$ with a $\sigma_{2}$ value of $49 \pm 15$ GM per chromophore. This spectrum appears to be narrower than its linear counterpart, which we attribute to the stronger influence of scattering on the latter. The spectral overlap between both spectra is due to the excitation of the lowest excited state of the molecule in both cases, in agreement with its symmetry. 

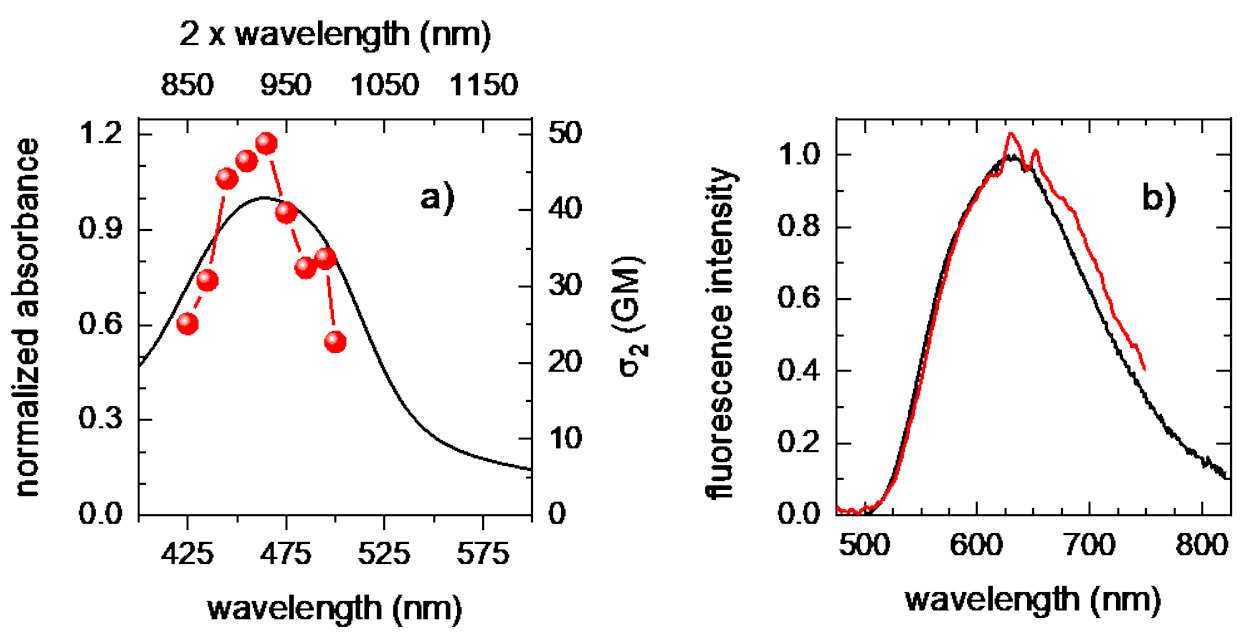

Figure 7 : a) Absorbance spectrum of aqueous solution of NP-I-PEG (black) superposed with their two-photon absorption spectrum per chromophore unit in the NP (red). b) One-(black) and two-photon (red) absorption induced fluorescence spectra of the NP-I-PEG.

The one and two-photon excitation induced fluorescence (TPEF) spectra exhibiting maxima at around $630 \mathrm{~nm}$ are in good agreement (Figure 7-b). The small discrepancy between the two spectra is probably due to the strong scattering of the exciting laser in the TPEF experiments. The overall NP brightness $\left(\phi_{\mathrm{f}} \times \sigma_{2}{ }^{\max } \times N_{N P}\right)$, which is the main parameter to demonstrate the potential of these objects for imaging applications, was calculated from the fluorescence quantum yield, the twophoton cross section and the estimated number of fluorophores per NP. ${ }^{56}$ This yields a very high value of (3.0 \pm 0.9$) \times 10^{6} \mathrm{GM}$ per NP, due to the very large number of dye molecules present in each NP (ca $2 \times 10^{6}$ fluorophores/NP in a $170 \mathrm{~nm}$ NP; see supporting information). ${ }^{36}$ This represents a significant advantage of these NP, since they will be imaged as a very bright spot, in contrast to the case of NPs where the dyes are diluted within a matrix.

To demonstrate the imaging potential of these nanoparticles, we first decided to study their ability to penetrate MCF-7 human breast cancer cells in culture and to emit luminescence. The biocompatibility of NP-I-PEG was firstly studied. The toxicity of NP-I-PEG on MCF-7 was investigated in the dark (for $72 \mathrm{~h}$ ) and exposed or not to sunlight (4 h). The results demonstrated a 
decrease in living cells according to the increase in NP-I-PEG concentration but no influence of light on cytotoxicity (Figure S11). Then, MCF-7 cells were incubated with NP-I-PEG (10 $\mu$ g.mL $\mathrm{mL}^{-}$ ${ }^{1}$ ) for $24 \mathrm{~h}$ and imaged using a confocal microscope equipped with a Chameleon pulsed laser. The brightness of NP-I-PEG inside living cells under $450 \mathrm{~nm}$ (continuous laser) or $900 \mathrm{~nm}$ (pulsed laser) excitation are compared in Figure S12. Cells were stained with a green CellMask revealing cells outline in green. Inside the cells, relatively weak red spots are observed under $450 \mathrm{~nm}$ excitation wavelength, suggesting that NPs are internalized within the cells. In contrast, under twophoton excitation (900 nm), the NP-I-PEG demonstrated a much higher brightness, resulting in the red coloration of all cells. The pictures displayed in Figure S12 demonstrate the significant potential of NP-I-PEG as fluorophores for bio-imaging under two-photon excitation in the NIR region.

In order to determine the sub-cellular localization of NP-I-PEG, confocal fluorescent imaging analysis was performed on living MCF-7 incubated with $25 \mu \mathrm{g} \cdot \mathrm{mL}^{-1}$ of NP-I-PEG in the presence of cell mask, Hoechst 33342 (nuclei staining), or LysoTracker (lysosomes staining). As shown in figure 8, at this concentration, the NP exhibit a tremendous brightness, mainly under an excitation at $900 \mathrm{~nm}$ using a pulsed laser. The first lane (cell membrane staining) confirms that the NPs are well inside the cells, the second one shows the perinuclear localization of the NPs, while the third one demonstrates a strong co-localization rate of NPs and lysosomes, suggesting an endolysosomal pathway for the internalization of NP-I-PEG inside the cancer cells studied here. 
$\lambda$ exc $=450 \mathrm{~nm}$

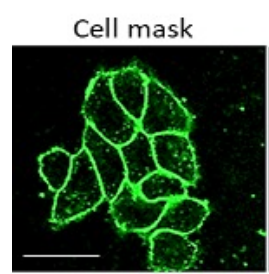

Hoechst

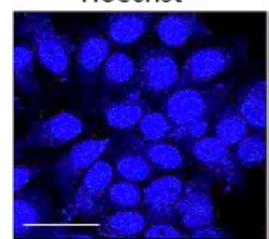

Lysotracker
NP-I-PEG

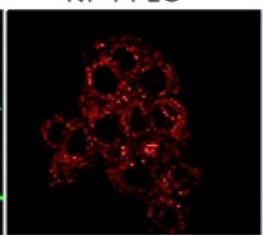

NP-I-PEG

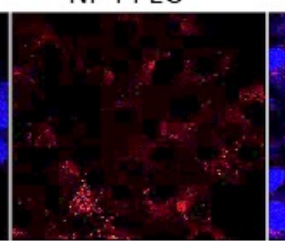

NP-I-PEG
Merged

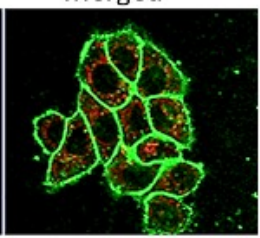

Merged

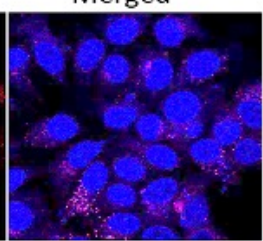

Merged

$\lambda$ exc $=900 \mathrm{~nm}$
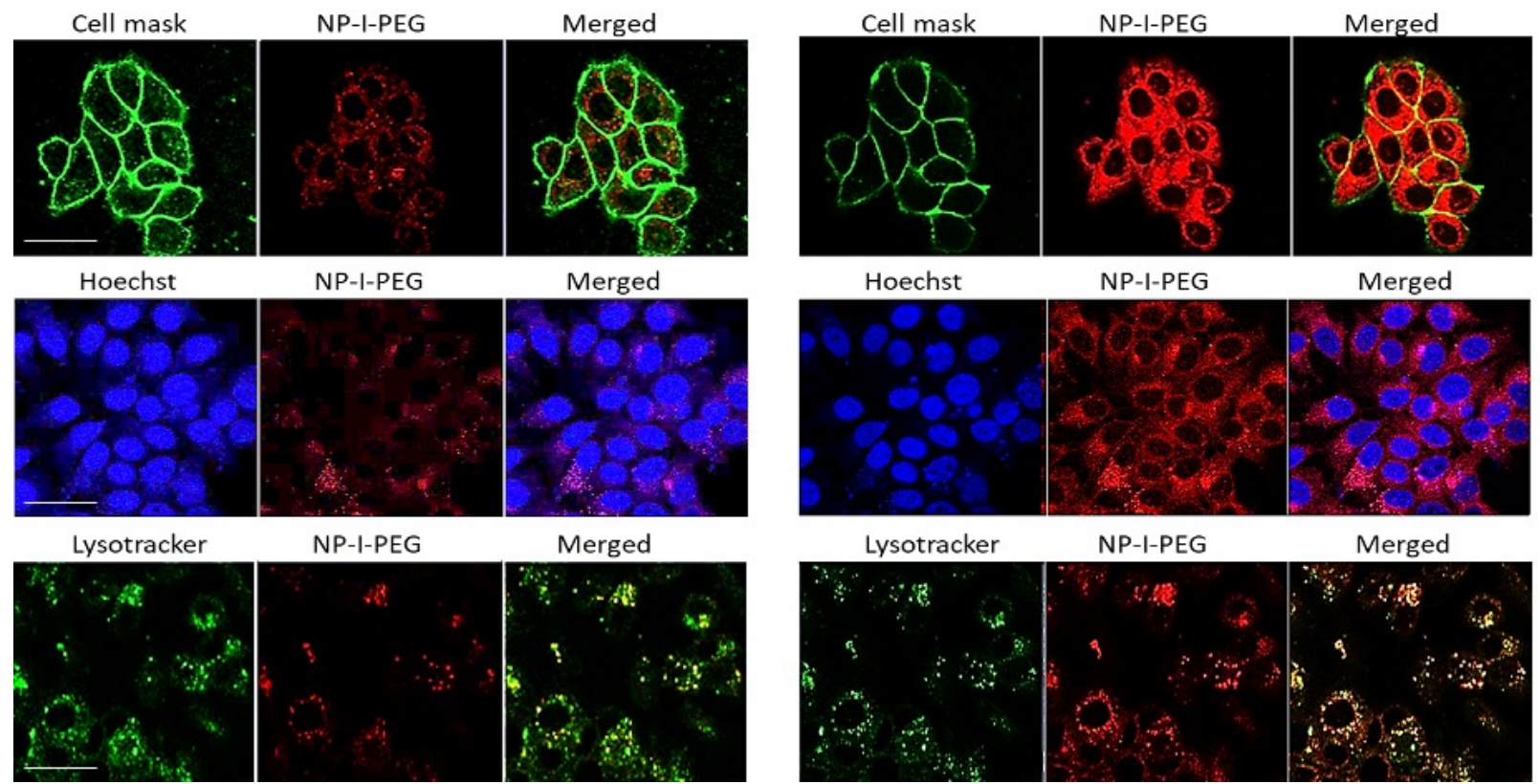

Lysotracker

NP-I-PEG

Merged

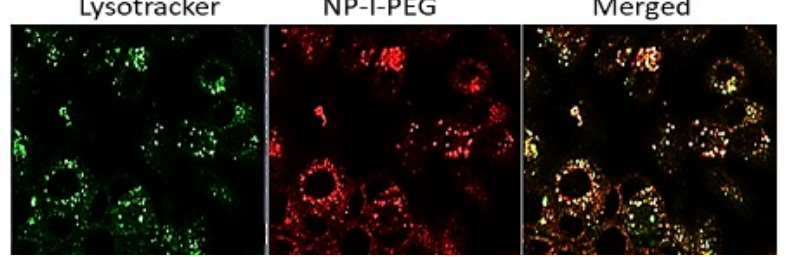

Figure 8: Subcellular localization of NP-I-PEG in living breast cancer cells (MCF-7). MCF 7 cells were incubated for 24 $h$ with $25 \mu \mathrm{g} \cdot \mathrm{mL}^{-1}$ of NP-I-PEG. Cells were washed twice and imaged using confocal microscope Zeiss LSM 780 laser beam at $450 \mathrm{~nm}$ or at $900 \mathrm{~nm}$ (pulsed laser, Chameleon), magnification $\times 63$. Cell membranes were stained with cell mask (green); nuclei were stained by Hoechst 33342 (blue); lysosomes were stained with LysoTracker green and compounds were visualized in red. Scale bar: $10 \mu \mathrm{m}$.

The potential for in vivo, two-photon fluorescence imaging was then probed on CX3CR1-GFP mice, expressing GFP only in microglia in the brain. Imaging was performed through the thinned skull using a two-photon microscope with a Ti:Sapphire laser operating at an excitation wavelength of $920 \mathrm{~nm}$. The optical background noise in the blood vessels of a CX3CR1-GFP mouse was first recorded prior to injection of NPs. Following this, $200 \mu \mathrm{L}$ of a NP-I-PEG suspension in PBS (80 $\mu \mathrm{g} / \mathrm{mL}$ NPs corresponding to $32 \mu \mathrm{g} / \mathrm{mL}$ dye), were introduced into the mouse through retroorbital injection. Imaging was performed within 30 seconds post injection, which enabled the visualization of the NPs in the blood vessels ( $\phi$ ca 20 and $40 \mu \mathrm{m}$ ), where they appeared as bright red spots in the red channel (Figure 9). Since the fluorescence intensity of the surrounding glial cells in the green channel did not evolve much with time, this was used as a reference to ascertain the fluorescence intensity of the circulating NPs at different time points. These red spots 
corresponding to NP varied in size and intensity, with some brighter than the others, which was attributed either to the presence of the NPs in the focal plane of the microscope or to the presence of a small percentage of aggregates of NPs, whose presence was demonstrated in the DLS studies in small observation volumes. Moreover, these bright NPs were observed to flow through the blood stream, without adhering to the walls of the blood vessels, an indication of their colloidal stability.

(a)

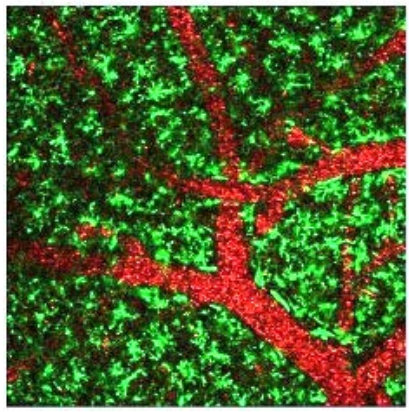

Figure 9: Circulation of NPs in blood vessels; (a) green channel (GFP microglia in the brain) and red channel (blood vessel due to presence of NP; b) red channel only. Image size $200 \times 200 \mu m^{2}$

A significant drop in fluorescence intensity (Figure 11-a) was recorded within 15-20 minutes of imaging, indicating the possible clearance of the biggest NPs and aggregates from the bloodstream (Figure 11-b). Although the functionalized NPs are colloidally stable in biological media, it has been reported that NPs larger than a few tens of nm are easily detected and cleared by the macrophages, while smaller NPs can circulate for longer periods of times. ${ }^{57}$ This further explains the scarce bright spots and faint fluorescence that is seen in the blood vessels even after a long time post injection, which could be due to the smaller sized NPs that are still circulating in the blood stream. It is obvious from Figure S13 that the fluorescence drops to a much lower signal within 5 minutes, but that some NPs still circulate the blood stream although fewer in number due to the elimination of the largest NPs.

However, even with this drop in fluorescence intensity, these bright spots were still visible even after 15 minutes post injection, indicating the circulation of the NPs in the bloodstream. This was 
further confirmed in Figure S13-b, where fluorescing NPs were still clearly visible after 15 minutes post injection.

\section{Conclusion}

We demonstrated here the potential of ultrabright silica-coated organic nanocrystals for bioimaging. These NPs containing crystalline emitters, specifically engineered to exhibit two-photon absorption and emission properties for two-photon bio-imaging. The NPs were functionalized with PEG chains (Mn: 5000), using CuAAC click chemistry to render them "stealthy" within the blood stream and improve their colloidal stability. The colloidal stability of the NPs was tested in different media with increasing levels of complexity starting with simulated body fluid (SBF) and different plasma proteins in increasing concentrations. The ACFs of NPs in SBF and varying concentrations of $\gamma$-globulins (10-45 mg/mL) demonstrated their colloidal stability, with no significant increase in mean size distribution being observed. A slight shift of the ACFs was observed towards longer decay times for high BSA concentrations (30 and $60 \mathrm{mg} / \mathrm{mL}$ ), indicating an increase in the solution polydispersity under these conditions. Finally, the colloidal stability of the functionalized NPs was investigated in an environment composed of a combination of individually tested components at their highest concentrations, ie in SBF with $60 \mathrm{mg} / \mathrm{mL}$ of BSA, $45 \mathrm{mg} / \mathrm{mL}$ of $\gamma$-globulin together with $1 \mathrm{mg} / \mathrm{mL}$ of glucose. Under such drastic conditions, no further aggregation was evident. These standard DLS results were further corroborated with small-volume DLS studies, which enabled the observation of a much smaller number of NPs thereby reducing the averaging aspect of standard DLS experiments. Spectroscopic characterization of the colloidal NP suspensions indicated a fluorescence quantum yield of $3 \%$ and also showed good agreement between the one and twophoton excitation induced fluorescence spectra peaking at $\sim 630 \mathrm{~nm}$, with a maximal $\sigma_{2}$ value of 
$50 \pm 15 \mathrm{GM}$ per molecule at $930 \mathrm{~nm}$. This results in a very high brightness per NP of (3.0+0.9) x $10^{6}$ GM. The potential of these NPs for imaging was validated in vitro in MCF-7 human breast cancer cells, and in vivo in CX3CR1-GFP mice, using two-photon imaging, where they appeared as bright red spots in the blood vessels. The NPs remained in circulation for several minutes post injection and without any adherence to the walls of the blood vessels, showing their potential for bio-imaging. Although a drop in fluorescence intensity was recorded after 15-20 minutes of imaging, corresponding to the clearance of the larger NPs by the reticulo-endothelial system, the smaller NPs remained in circulation and were detectable. These promising results show the potential of these unique nanotracers for bio-imaging applications. Studies are underway to further reduce the size distribution of the NPs to achieve longer circulation times.

\section{Acknowledgements}

A.B., G.M. and Y.B. acknowledge the contribution of SFR Biosciences (UMS3444/CNRS, US8/Inserm, ENS de Lyon, UCBL) facilities PLATIM, for access to the femtosecond laser system and for the technical assistance.

M.G.B. and C.N acknowledge the imaging facility MRI, member of the national infrastructure France-BioImaging supported by the French National Research Agency (ANR-10-INBS-04, «Investments for the future»).

The authors thanks O. Stephan (Liphy laboratory, UMR5588/CNRS-UGA Grenoble) for the utilization of the commercial Malvern Zetasizer Nano ZS apparatus and B. Van Der Sanden (TIMC-IMAG Lab) for helpful discussions. Prof J. R. Bartlett (Western Sydney University) is warmly acknowledged for carefully reading this manuscript.

Supporting Information Available: DLS and FCS mathematical model used, IR and DLS characterizations. This material is available free of charge via the Internet at http://pubs.acs.org. 


\section{References}

(1) Choy, G.; Choyke, P.; Libutti, S. K. Current Advances in Molecular Imaging: Noninvasive in Vivo Bioluminescent and Fluorescent Optical Imaging in Cancer Research. Mol Imaging 2003, 2 (4), 303-312.

(2) Hadjipanayis, C.; Jiang, H.; Roberts, D.; Yang, L. Current and Future Clinical Applications for Optical Imaging of Cancer: From Intraoperative Surgical Guidance to Cancer Screening. Semin. Oncol. 2011, 38 (1), 109-118. https://doi.org/10.1053/j.seminoncol.2010.11.008.Current.

(3) Pierce, M.; Javier, D.; Richards-Kortum, R. Optical Contrast Agents and Imaging Systems for Detection and Diagnosis of Cancer. Int J Cancer 2008, 123 (9), 1979-1990. https://doi.org/10.1002/ijc.23858.Optical.

(4) Luker, G. D.; Luker, K. E. Optical Imaging: Current Applications and Future Directions. J. Nucl. Med. 2007, 49 (1), 1-4. https://doi.org/10.2967/jnumed.107.045799.

(5) Sun, P.; Wu, Q.; Sun, X.; Miao, H.; Deng, W.; Zhang, W.; Fan, Q.; Huang, W. J-Aggregate Squaraine Nanoparticles with Bright NIR-II Fluorescence for Imaging Guided Photothermal Therapy. Chem. Commun. 2018, 54 (95), 13395-13398. https://doi.org/10.1039/c8cc08096h.

(6) Thomas, G.; Van Voskuilen, J.; Gerritsen, H. C.; Sterenborg, H. J. C. M. Advances and Challenges in Label-Free Nonlinear Optical Imaging Using Two-Photon Excitation Fluorescence and Second Harmonic Generation for Cancer Research. J. Photochem. Photobiol. B Biol. 2014, 141, 128-138. https://doi.org/10.1016/j.jphotobiol.2014.08.025.

(7) Hemmer, E.; Benayas, A.; Légaré, F.; Vetrone, F. Exploiting the Biological Windows: Current Perspectives on Fluorescent Bioprobes Emitting above $1000 \mathrm{Nm}$. Nanoscale Horizons 2016, 1 (3), 168-184. https://doi.org/10.1039/c5nh00073d.

(8) Svoboda, K.; Yasuda, R. Principles of Two-Photon Excitation Microscopy and Its Applications to Neuroscience. Neuron 2006, 50 (6), 823-839. https://doi.org/10.1016/j.neuron.2006.05.019.

(9) Würthner, F.; Kaiser, T. E.; Saha-Möller, C. R. J-Aggregates: From Serendipitous Discovery to Supramolecular Engineering of Functional Dye Materials. Angew. Chemie - Int. Ed. 2011, 50 (15), 3376-3410. https://doi.org/10.1002/anie.201002307.

(10) Chen, W.; Cheng, C.-A.; D. Cosco, E.; Ramakrishnan, S.; G. P.Lingg, J.; T. Bruns, O.; I. Zink, J.; M. Sletten, E. Shortwave Infrared Imaging with J-Aggregates Stabilized in Hollow Mesoporous Silica Nanoparticles. J Am Chem Soc. 2019, 141 (32) (5), 12475-12480. https://doi.org/10.1016/j.physbeh.2017.03.040.

(11) Song, X.; Gong, H.; Liu, T.; Cheng, L.; Wang, C.; Sun, X.; Liang, C.; Liu, Z. J-Aggregates of 
Organic Dye Molecules Complexed with Iron Oxide Nanoparticles for Imaging-Guided Photothermal Therapy under 915-Nm Light. Small 2014, 10 (21), 4362-4370.

https://doi.org/10.1002/smll.201401025.

(12) Kaiser, T. E.; Wang, H.; Stepanenko, V.; Würthner, F. Supramolecular Construction of Fluorescent J-Aggregates Based on Hydrogen-Bonded Perylene Dyes. Angew. Chemie Int. Ed. 2007, 46 (29), 5541-5544. https://doi.org/10.1002/anie.200701139.

(13) Yang, C.; Wang, X.; Wang, M.; Xu, K.; Xu, C. Robust Colloidal Nanoparticles of Pyrrolopyrrole Cyanine J-Aggregates with Bright Near-Infrared Fluorescence in Aqueous Media: From Spectral Tailoring to Bioimaging Applications. Chem. - A Eur. J. 2017, 23 (18), 4310-4319. https://doi.org/10.1002/chem.201604741.

(14) Song, X.; Zhang, R.; Liang, C.; Chen, Q.; Gong, H.; Liu, Z. Nano-Assemblies of J-Aggregates Based on a NIR Dye as a Multifunctional Drug Carrier for Combination Cancer Therapy. Biomaterials 2015, 57, 84-92. https://doi.org/10.1016/j.biomaterials.2015.04.001.

(15) Biswas, S.; Ahn, H. Y.; Bondar, M. V.; Belfield, K. D. Two-Photon Absorption Enhancement of Polymer-Templated Porphyrin-Based J-Aggregates. Langmuir 2012, 28 (2), 1515-1522. https://doi.org/10.1021/la203883k.

(16) Gurunatha, K. L.; Dujardin, E. Tuning the Optical Coupling between Molecular Dyes and Metal Nanoparticles by the Templated Silica Mineralization of J-Aggregates. J. Phys. Chem. C 2013, 117 (7), 3489-3496. https://doi.org/10.1021/jp311911f.

(17) Shakiba, M.; Ng, K. K.; Huynh, E.; Chan, H.; Charron, D. M.; Chen, J.; Muhanna, N.; Foster, F. S.; Wilson, B. C.; Zheng, G. Stable J-Aggregation Enabled Dual Photoacoustic and Fluorescence Nanoparticles for Intraoperative Cancer Imaging. Nanoscale 2016, 8 (25), 12618-12625. https://doi.org/10.1039/c5nr08165c.

(18) Qin, W.; Ding, D.; Liu, J.; Yuan, W. Z.; Hu, Y.; Liu, B.; Tang, B. Z. Biocompatible Nanoparticles with Aggregation-Induced Emission Characteristics as Far-Red/nearInfrared Fluorescent Bioprobes for in Vitro and in Vivo Imaging Applications. Adv. Funct. Mater. 2012, 22 (4), 771-779. https://doi.org/10.1002/adfm.201102191.

(19) Geng, J.; Li, K.; Ding, D.; Zhang, X.; Qin, W.; Liu, J.; Tang, B. Z.; Liu, B. Lipid-PEG-Folate Encapsulated Nanoparticles with Aggregation Induced Emission Characteristics: Cellular Uptake Mechanism and Two-Photon Fluorescence Imaging. Small 2012, 8 (23), 36553663. https://doi.org/10.1002/smll.201200814.

(20) Montalti, M.; Prodi, L.; Rampazzo, E.; Zaccheroni, N. Dye-Doped Silica Nanoparticles as Luminescent Organized Systems for Nanomedicine. Chem. Soc. Rev. 2014, 43 (12), 42434268. https://doi.org/10.1039/C3CS60433K.

(21) Rampazzo, E.; Genovese, D.; Palomba, F.; Prodi, L.; Zaccheroni, N. NIR Fluorescent Dye Doped Silica Nanoparticles for in Vivo Imaging, Sensing and Theranostic. Methods Appl. 
Fluoresc. 2018, 6, 022002. https://doi.org/10.1088/2050-6120/aa8f57.

(22) Bonacchi, S.; Genovese, D.; Juris, R.; Montalti, M.; Prodi, L.; Rampazzo, E.; Zaccheroni, N. Luminescent Silica Nanoparticles: Extending the Frontiers of Brightness. Angew. Chemie Int. Ed. 2011, 50 (18), 4056-4066. https://doi.org/10.1002/anie.201004996.

(23) Hanske, C.; Sanz-Ortiz, M. N.; Liz-Marzán, L. M. Silica-Coated Plasmonic Metal Nanoparticles in Action. Adv. Mater. 2018, 30 (27), 1707003.

https://doi.org/10.1002/adma.201707003.

(24) Selvan, S. T.; Tan, T. T.; Ying, J. Y. Robust, Non-Cytotoxic, Silica-Coated CdSe Quantum Dots with Efficient Photoluminescence. Adv. Mater. 2005, 17 (13), 1620-1625. https://doi.org/10.1002/adma.200401960.

(25) Ang, E. H.; Zeng, J.; Subramanian, G. S.; Chellappan, V.; Sudhaharan, T.; Padmanabhan, P.; Gulyás, B.; Tamil Selvan, S. Silica-Coated Mn-Doped ZnS Nanocrystals for Cancer Theranostics. ACS Appl. Nano Mater. 2020, 3 (3), 3088-3096. https://doi.org/10.1021/acsanm.0c00598.

(26) Wang, Y.-F.; Che, J.; Zheng, Y.-C.; Zhao, Y.-Y.; Chen, F.; Jin, S.-B.; Gong, N.-Q.; Xu, J.; Hu, Z.B.; Liang, X.-J. Multi-Stable Fluorescent Silica Nanoparticles Obtained from in Situ Doping with Aggregation-Induced Emission Molecules. J. Mater. Chem. B 2015, 3 (45), 87758781. https://doi.org/10.1039/C5TB01761K.

(27) Wang, X.; Song, P.; Peng, L.; Tong, A.; Xiang, Y. Aggregation-Induced Emission LuminogenEmbedded Silica Nanoparticles Containing DNA Aptamers for Targeted Cell Imaging. ACS Appl. Mater. Interfaces 2016, 8 (1), 609-616. https://doi.org/10.1021/acsami.5b09644.

(28) Li, Q.; Qian, Y. Aggregation-Induced Emission Enhancement and Cell Imaging of a Novel (Carbazol-N-YI)Triphenylamine-BODIPY. New J. Chem. 2016, 40 (8), 7095-7101. https://doi.org/10.1039/C6NJ01495J.

(29) Wei, Y.; Liu, M.; Xu, D.; Wan, Q.; Huang, Q.; Jiang, R.; Shi, Y.; Deng, F.; Zhang, X. Synthesis, Surface Modification and Biological Imaging of Aggregation-Induced Emission (AIE) Dye Doped Silica Nanoparticles. Appl. Surf. Sci. 2017, 403, 396-402. https://doi.org/10.1016/j.apsusc.2017.01.234.

(30) Kim, S.; Pudavar, H. E.; Bonoiu, A.; Prasad, P. N. Aggregation-Enhanced Fluorescence in Organically Modified Silica Nanoparticles: A Novel Approach toward High-Signal-Output Nanoprobes for Two-Photon Fluorescence Bioimaging. Adv. Mater. 2007, 19 (22), 37913795. https://doi.org/10.1002/adma.200700098.

(31) Kim, S.; Ohulchanskyy, T. Y.; Pudavar, H. E.; Pandey, R. K.; Prasad, P. N. Organically Modified Silica Nanoparticles Co-Encapsulating Photosensitizing Drug and AggregationEnhanced Two-Photon Absorbing Fluorescent Dye Aggregates for Two-Photon Photodynamic Therapy. J. Am. Chem. Soc. 2007, 129 (9), 2669-2675. 
https://doi.org/10.1021/ja0680257.

(32) Philippot, C.; Dubois, F.; Bacia, M.; Djurado, E.; Ibanez, A. Fluorescent Organic Nanocrystal Confined in Sol-Gel Matrix for Bio-Imaging. J. Sol-Gel Sci. Technol. 2011, 57 (3), 253-257. https://doi.org/10.1007/s10971-010-2175-z.

(33) Dubuisson, E.; Szunerits, S.; Bacia, M.; Pansu, R.; Ibanez, A. Fluorescent Molecular Nanocrystals Anchored in Sol-Gel Thin Films: A Label-Free Signalization Function for Biosensing Applications. New J. Chem. 2011, 35 (11), 2416-2421. https://doi.org/10.1039/C1NJ20353C.

(34) Ibanez, A.; Maximov, S.; Guiu, A.; Chaillout, C.; Baldeck, P. L. Controlled Nanocrystallization of Organic Molecules in Sol-Gel Glasses. Adv. Mater. 1998, 10 (18), 1540-1543. https://doi.org/10.1002/(SICI)1521-4095(199812)10:18<1540::AIDADMA1540>3.0.CO;2-1.

(35) Monnier, V.; Sanz, N.; Botzung-Appert, E.; Bacia, M.; Ibanez, A. Confined Nucleation and Growth of Organic Nanocrystals in Sol-Gel Matrices. J. Mater. Chem. 2006, 16 (15), 14011409. https://doi.org/10.1039/B509833P.

(36) Shenoi-Perdoor, S.; Cattoën, X.; Bretonnière, Y.; Eucat, G.; Andraud, C.; Gennaro, B.; Kodjikian, S.; Dubois, F.; Ibanez, A. Red-Emitting Fluorescent Organic@silicate Core-Shell Nanoparticles for Bio-Imaging. New J. Chem. 2018, 42 (18), 15353-15360. https://doi.org/10.1039/C8NJ03297A.

(37) Philippot, C.; Dubois, F.; Maurin, M.; Boury, B.; Prat, A.; Ibanez, A. New Core-Shell Hybrid Nanoparticles for Biophotonics: Fluorescent Organic Nanocrystals Confined in Organosilicate Spheres. J. Mater. Chem. 2012, 22 (22), 11370. https://doi.org/10.1039/c2jm30875d.

(38) Philippot, C.; Zimmermann, J.; Dubois, F.; Bacia, M.; Boury, B.; Baldeck, P. L.; Brasselet, S.; Ibanez, A. Polymorphism of CMONS Nanocrystals Grown in Silicate Particles through a Spray-Drying Process. Cryst. Growth Des. 2013, 13 (12), 5241-5248. https://doi.org/10.1021/cg401000t.

(39) Redon, S.; Eucat, G.; Ipuy, M.; Jeanneau, E.; Gautier-Luneau, I.; Ibanez, A.; Andraud, C.; Bretonnière, Y. Tuning the Solid-State Emission of Small Push-Pull Dipolar Dyes to the FarRed through Variation of the Electron-Acceptor Group. Dye. Pigment. 2018, 156 (March), 116-132. https://doi.org/10.1016/j.dyepig.2018.03.049.

(40) Shenoi-Perdoor, S.; Noureddine, A.; Dubois, F.; Wong Chi Man, M.; Cattoën, X. Click Functionalization of Sol-Gel Materials. In Handbook of Sol-Gel Science and Technology: Processing, Characterization and Applications; Klein, L., Aparicio, M., Jitianu, A., Eds.; Springer International Publishing: Cham, 2018; pp 3001-3040. https://doi.org/10.1007/978-3-319-32101-1_95. 
(41) Ma, K.; Zhang, D.; Cong, Y.; Wiesner, U. Elucidating the Mechanism of Silica Nanoparticle PEGylation Processes Using Fluorescence Correlation Spectroscopies. Chem. Mater. 2016, 28 (5), 1537-1545. https://doi.org/10.1021/acs.chemmater.6b00030.

(42) Rio-Echevarria, I. M.; Selvestrel, F.; Segat, D.; Guarino, G.; Tavano, R.; Causin, V.; Reddi, E.; Papini, E.; Mancin, F. Highly PEGylated Silica Nanoparticles: "Ready to Use" Stealth Functional Nanocarriers. J. Mater. Chem. 2010, 20 (14), 2780. https://doi.org/10.1039/b921735e.

(43) Cauda, V.; Argyo, C.; Bein, T. Impact of Different PEGylation Patterns on the Long-Term Bio-Stability of Colloidal Mesoporous Silica Nanoparticles. J. Mater. Chem. 2010, 20 (39), 8693. https://doi.org/10.1039/c0jm01390k.

(44) Adumeau, L.; Genevois, C.; Roudier, L.; Schatz, C.; Couillaud, F.; Mornet, S. Impact of Surface Grafting Density of PEG Macromolecules on Dually Fluorescent Silica Nanoparticles Used for the in Vivo Imaging of Subcutaneous Tumors. Biochim. Biophys. Acta - Gen. Subj. 2017, 1861 (6), 1587-1596. https://doi.org/10.1016/j.bbagen.2017.01.036.

(45) Şen Karaman, D.; Gulin-Sarfraz, T.; Hedström, G.; Duchanoy, A.; Eklund, P.; Rosenholm, J. M. Rational Evaluation of the Utilization of PEG-PEI Copolymers for the Facilitation of Silica Nanoparticulate Systems in Biomedical Applications. J. Colloid Interface Sci. 2014, 418, 300-310. https://doi.org/10.1016/j.jcis.2013.11.080.

(46) Nissinen, T.; Näkki, S.; Laakso, H.; Kučiauskas, D.; Kaupinis, A.; Kettunen, M. I.; Liimatainen, T.; Hyvönen, M.; Valius, M.; Gröhn, O.; Lehto, V. P. Tailored Dual PEGylation of Inorganic Porous Nanocarriers for Extremely Long Blood Circulation in Vivo. ACS Appl. Mater. Interfaces 2016, 8 (48), 32723-32731. https://doi.org/10.1021/acsami.6b12481.

(47) He, Q.; Zhang, J.; Shi, J.; Zhu, Z.; Zhang, L.; Bu, W.; Guo, L.; Chen, Y. The Effect of PEGylation of Mesoporous Silica Nanoparticles on Nonspecific Binding of Serum Proteins and Cellular Responses. Biomaterials 2010, 31 (6), 1085-1092.

https://doi.org/10.1016/j.biomaterials.2009.10.046.

(48) Moitra, N.; Trens, P.; Raehm, L.; Durand, J.-O.; Cattoën, X.; Wong Chi Man, M. Facile Route to Functionalized Mesoporous Silica Nanoparticles by Click Chemistry. J. Mater. Chem. 2011, 21 (35), 13476-13482. https://doi.org/10.1039/c1jm12066b.

(49) Xu, C.; Webb, W. W. Measurement of Two-Photon Excitation Cross Sections of Molecular Fluorophores with Data from 690 to 1050 Nm. J. Opt. Soc. Am. B 1996, 13 (3), 481-491. https://doi.org/10.1364/JOSAB.13.000481.

(50) de Reguardati, S.; Pahapill, J.; Mikhailov, A.; Stepanenko, Y.; Rebane, A. High-Accuracy Reference Standards for Two-Photon Absorption in the 680\&\#x2013;1050 Nm Wavelength Range. Opt. Express 2016, 24 (8), 9053-9066.

https://doi.org/10.1364/OE.24.009053. 
(51) Bürglová, K.; Noureddine, A.; Hodačová, J.; Toquer, G.; Cattoën, X.; Wong Chi Man, M. A General Method for Preparing Bridged Organosilanes with Pendant Functional Groups and Functional Mesoporous Organosilicas. Chem. Eur. J. 2014, 20 (33), 10371-10382. https://doi.org/10.1002/chem.201403136.

(52) Barbara, A.; Dubois, F.; Ibanez, A.; Eng, L. M.; Quémerais, P. SERS Correlation Spectroscopy of Silver Aggregates in Colloidal Suspension: Quantitative Sizing Down to a Single Nanoparticle. J. Phys. Chem. C 2014, 118 (31), 17922-17931. https://doi.org/10.1021/jp5024444.

(53) Hosokawa, C.; Yoshikawa, H.; Masuhara, H. Cluster Formation of Nanoparticles in an Optical Trap Studied by Fluorescence Correlation Spectroscopy. Phys. Rev. E 2005, 72 (2), 21408. https://doi.org/10.1103/PhysRevE.72.021408.

(54) Ke, P. C.; Lin, S.; Parak, W. J.; Davis, T. P.; Caruso, F. A Decade of the Protein Corona. ACS Nano 2017, 11 (12), 11773-11776. https://doi.org/10.1021/acsnano.7b08008.

(55) Adamczyk, Z.; Sadlej, K.; Wajnryb, E.; Ekiel-Jeżewska, M. L.; Warszyński, P. Hydrodynamic Radii and Diffusion Coefficients of Particle Aggregates Derived from the Bead Model. J. Colloid Interface Sci. 2010, 347 (2), 192-201. https://doi.org/https://doi.org/10.1016/j.jcis.2010.03.066.

(56) Pawlicki, M.; Collins, H. A.; Denning, R. G.; Anderson, H. L. Two-Photon Absorption and the Design of Two-Photon Dyes. Angew. Chemie Int. Ed. 2009, 48 (18), 3244-3266. https://doi.org/10.1002/anie.200805257.

(57) Chou, L. Y. T.; Chan, W. C. W. Fluorescence-Tagged Gold Nanoparticles for Rapidly Characterizing the Size-Dependent Biodistribution in Tumor Models. Adv. Healthc. Mater. 2012, 1 (6), 714-721. https://doi.org/10.1002/adhm.201200084.

\section{SYNOPSIS}




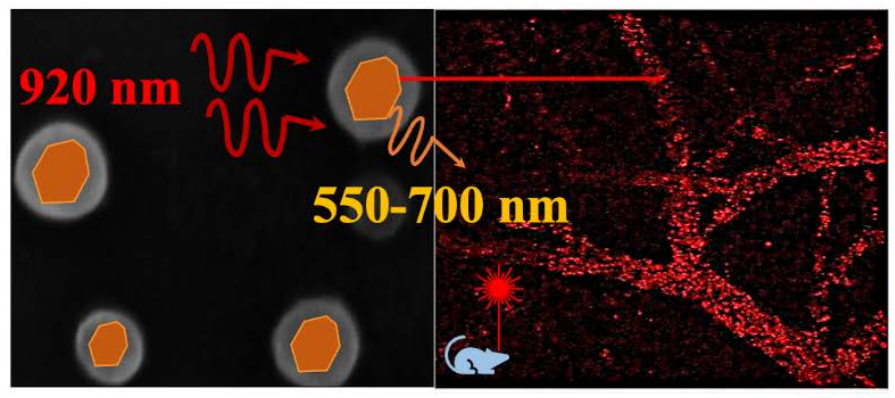

